\title{
Adjunctive Platelet-Rich Plasma (PRP) in Infrabony Regenerative Treatment: A Systematic Review and RCT's Meta-Analysis
}

\author{
Mubashir Saleem, ${ }^{1}$ Flavio Pisani ${ }^{1},{ }^{1}$ Faisal Maqbool Zahid, ${ }^{2}$ Ioannis Georgakopoulos, ${ }^{3}$ \\ Teuta Pustina-Krasniqi $\left(\mathbb{D},{ }^{4}\right.$ Edit Xhajanka, ${ }^{5}$ and Maher Almasri ${ }^{1}$ \\ ${ }^{1}$ Faculty of Dentistry, School of Health, MClinDent in Periodontology, BPP University Birmingham, Birmingham, UK \\ ${ }^{2}$ Department of Statistics, Ludwig-Maximilians-University Munich, Munich, Germany \\ ${ }^{3}$ World Academy of Growth Factors and Stem Cells in Dentistry, Athens, Greece \\ ${ }^{4}$ Universiteti I Prishtines "Hasan Prishtina", Prishtine, Kosovo \\ ${ }^{5}$ Universiteti I Mjekesise Dentare, Tirane, Albania
}

Correspondence should be addressed to Flavio Pisani; flaviopisani@bpp.com

Received 25 November 2017; Accepted 11 January 2018; Published 19 March 2018

Academic Editor: Marc L. Turner

Copyright (c) 2018 Mubashir Saleem et al. This is an open access article distributed under the Creative Commons Attribution License, which permits unrestricted use, distribution, and reproduction in any medium, provided the original work is properly cited.

\begin{abstract}
Background and Objective. The purpose of this study was to highlight the clinical performance of platelet-rich plasma (PRP) used as an adjunctive tool for regeneration in infrabony periodontal defects using different biomaterials or performing different surgical flap approaches. Comparative evaluation of main clinical outcomes as probing pocket depth reduction, clinical attachment gain, and recession reduction with and without the use of PRP has been analysed. Materials and Methods. According to the focused question, an electronic and hand searching has been performed up to December 2016. From a batch of 73 articles, the selection strategy and Jadad quality assessment led us to include 15 studies for the meta-analysis. Results. Despite the high heterogeneity found and the lack of complete data regarding the selected clinical outcomes, a comparative analysis has been possible by the categorization of used biomaterials and surgical flap approaches. This method led us to observe the best performance of grafts with the use of adjunctive PRP in CAL gain and PPD reduction. No difference has been outlined with a specific surgical flap. Conclusions. Although PRP is considered a cheap and patient's derived growth factor, the not conclusive data reported would suggest that its use in addition to bone substitutes could be of some clinical benefit in the regenerative treatment of infrabony defects. Clinical Relevance. This systematic review was intended to sort out the huge controversial debate in the field about the possible use of PRP in regenerative surgery in infrabony defect. The clinical relevance of using blood-borne growth factors to conventional procedures is effective as these could determine a better performance and outcomes despite the surgical approach adopted and limit the use of additional biomaterials for the blood clot stabilization.
\end{abstract}

\section{Introduction}

The ultimate goal of periodontal therapy in the case of infrabony defects is regeneration. Regeneration means "reproduction or reconstitution of a lost or injured part. It takes into account all the procedures attempting to regenerate lost periodontal structures through differential tissue responses and by different biomaterials such as grafts, membranes or Biomodulators as Enamel Matrix Proteins" (Glossary of Periodontal Terms, AAP, 2001). These procedures would temporarily delay the apical migration of the gingival epithelium allowing the granulation tissue derived from the periodontal ligament and osseous tissue to repopulate the space adjacent to the denuded root surface [1].

Several materials are available in daily practice, but none can be considered as an ideal one. In order to achieve a successful regeneration, biomaterials should fulfil four main characteristics: (1) structural integrity, (2) to work as a scaffold for tissue ingrowth, (3) favoured by stem cells that can potentially differentiate and support 
the regeneration, and (4) contain factors for regeneration and/or repair.

Growth factors (GFs) are expressed during different phases of healing, and they are key elements in promoting regeneration of tissues; these are considered the most relevant factors in osseoregenerative process.

Platelet-rich plasma (PRP) is considered a cheap way to obtain many growth factors (GFs) in physiological proportion, and it has already been largely applied as a GF's carrier in different tissues due to its properties of inducing healing response even in cases with low potential [2].

Substantially, PRP is a blood derivate growth factor with a higher platelet concentration up to $338 \%$ that can release all polypeptide GFs from alpha granules: plateletderived growth factor (PDGF), transforming growth factor- $\beta 1$ and transforming growth factor- $\beta 2$ (TGF- $\beta 1$ and TGF- $\beta 2$ ), and insulin-like growth factors 1 and 2 (IGF1,2) [3].

In clinical dental practice, the effective use of PRP has been described in sinus grafting procedures [4], alveolar socket preservation techniques [5], and also as an adjunctive procedure to support the regenerative process in periodontal infrabony and furcation defects [6].

Although its clinical benefits have been demonstrated several times, the adjunctive use of autologous PRP in regenerative procedures has produced controversial outcomes ranging from significant to null effects, as demonstrated from different published systematic reviews $[3,7]$.

This review investigates and updates the clinical efficacy of PRP when added to grafting materials and/or to membranes or biomodulators in cases of periodontal infrabony defects in patients with advanced chronic periodontitis. In particular, it was aimed to highlight the most relevant clinical outcome changes (vertical pocket probing depth, vertical clinical attachment level, and the recession) in GTR coupled with PRP compared to the same procedure without it. In order to get stronger evidence, a SR on published RCTs was chosen.

\section{Materials and Methods}

2.1. Focused Question. The focused question that this systematic review is intending to answer is

"What are the Vertical Probing Pocket Depth Reductions, the Vertical Clinical Attachment Level Gains and the Recession Reduction at infra-bony defects at least 6 months after Regenerative Surgery with the adjunctive use of PRP as documented in Randomized Clinical Trials, compared to the same clinical procedures and biomaterials performed without the use of PRP?"

2.2. Eligibility Criteria for Studies to be Included in This Review. According to the P.I.C.O system [8], inclusion criteria were outlined as follows:

2.2.1. Study Population. Studies were limited to human subjects older than 18 years and in good general health, with a diagnosis of chronic periodontitis and with at least one pair of specular infrabony defects. Studies considering individuals with a history of aggressive periodontitis or conducted on animal models were excluded from our consideration.

2.2.2. Type of Interventions. GTR surgical procedures with and without PRP will be the interventions considered for the comparative evaluation. The specific regenerative techniques and biomaterials investigated in this study were

(1) autologous bone grafts,

(2) bone substitutes (allogenic, xenogenic, and synthetic grafts),

(3) barrier membranes (resorbable and not resorbable),

(4) enamel matrix proteins (EMD).

2.2.3. Type of Comparison. Infrabony defects treated by regenerative surgery with PRP were considered the test group and compared to the same defects treated by the same regenerative therapy without PRP that were considered the control group.

2.2.4. Outcome Measures. Outcome variables considered in this study were

(i) probing pocket depth reduction (PPDRed $\mathrm{mm}$ ),

(ii) clinical attachment level gain (CALGain mm),

(iii) recession reduction (RECRed).

These were evaluated as the mean difference $(\mathrm{mm})$ from the time of surgery until the end of the evaluation period not before 6 months.

2.2.5. Types of Studies. To be considered for inclusion in this review, studies should be randomized controlled clinical trials (RCTs) only; no cohort studies (CHT) or case-control studies were included. Case series and case reports studies were also not considered as they would provide a low strength of evidence.

2.3. Information Sources. The search has been performed by the use of the following electronic databases: Pubmed, Cochrane Oral Library, Embase, and LILACS.

Trial registers have been searched using Current Controlled Trials (http://www.controlled-trials.com/), ClinicalTrials.gov (http://clinicalTrials.gov/ct2/home) and the World Health Organization International Trials Registry Platform search portal (http://www.who.int/trialsearch/ Default.aspx).

Conference abstracts have been searched using the ISI Web of knowledge (http://isiwebofknowledge.com) and the Grey literature using Open Grey (http://www.opengrey.eu).

Hand searching included a complete search of Journal of Periodontology and Journal of Clinical Periodontology up to December 2016 and bibliographies of all relevant papers and review articles. In the case of ambiguous or missing data, experts have been contacted directly.

The search has been performed up to and including December 2016. 


\section{Search Strategy}

The following search strategy has been used as a combination of $\mathrm{MeSH}$ terms and free text words:

(i) Intervention and materials: "PRP" [txt words] OR "Platelet Rich Plasma" [txt words] OR "Platelet" [mesh] OR "guided tissue regeneration" [mesh] OR "periodontal" [all fields] OR "regeneration [mesh] OR "guided-tissue-regeneration" [txt words] OR "GTR" [txt words] OR “periodontal regeneration" [txt words] OR "Bone filler" [txt words] OR "Autologous bone grafts" [txt words] OR "Autogenous bone" [txt words] OR "bone substitutes" [mesh] OR “allogenic grafts" [txt words] OR “Allografts" [txt words] OR "xenogenic grafts" [txt words] OR "xenografts" [mesh] OR "synthetic grafts" [txt words] OR "Barrier membranes" [txt words] OR "membranes" [mesh] OR "resorbable membrane" [txt words] OR "non-resorbable membrane" [txt words] OR "guided bone regeneration" [txt words] OR "GBR" [txt words] OR "freeze dried bone allograft" [txt words] OR "demineralized freeze dried bone allograft" [txt words] OR "DFDBA" [txt words] OR "FDBA" [txt words] OR "Bio-Oss" [txt words] OR "Bio-Oss Collagen" [txt words] OR "Alloplast" [txt words] OR "tricalciumphosphate" [txt words] OR “cerasorb” [txt words] OR "Bioglass" [txt words] OR "polymeric" [txt words] OR "collagen sponge" [txt words] OR "Collagen" [txt words] OR "Biogide" [txt words] OR "Ossix" [txt words] OR "Gore tex" [txt words] OR "Enamel Matrix Proteins" [txt words] OR "Emdogain" [txt words] OR “EMD” [txt words]);

(ii) Disease: ("periodontal defects" [mesh] OR "periodontal [all fields] OR "infrabony defects" [txt words]);

(iii) Study design: (."randomized clinical trials" [mesh] OR randomized controlled study" [mesh] OR "clinical trial" [mesh] OR "cohort study" [mesh] OR "clinical trial” [mesh] OR "comparative study" [mesh] OR "systematic review" [mesh] OR "case control study" [mesh] OR "longitudinal study" [mesh]).

Adopted filters have been "humans," and articles were published in English language.

\subsection{Methods of the Review}

3.1.1. Screening and Selection. Initially, titles and abstracts of all reports were screened independently by two reviewers (MS and FP). Subsequently, for studies appearing to meet the inclusion criteria, or for which there were insufficient data in the title and abstract to make a clear decision, the full report was obtained and independently assessed by three reviewers (MS, FP, and MA) to establish whether the study met the inclusion criteria. Any disagreements were resolved by discussion among the reviewers. All studies meeting the inclusion criteria then underwent validity assessment. The reasons for rejecting studies at this or at subsequent stages were recorded.

Special attention was paid not to duplicate publications in order to avoid a likely bigger impact of the same data in the global result.

3.1.2. Quality Assessment/Risk of Bias. The quality assessment of the included studies was undertaken independently by two reviewers based on the content of the articles. The reviewers were blind to the name of the authors, institutions, and journal titles.

A commonly used three-item, five-point quality scale was used to rate the quality of the studies [9]. The minimum score for the inclusion was 2 , and the maximum was 5 .

Points were awarded according to the following criteria:

(1) Was the study randomized? If yes, +1 point.

Was the randomization procedure appropriate and clearly reported in the study? If yes, +1 point. If no, all points deleted.

(2) Was the study double-blinded? If yes, +1 point.

Was the double-blinding method appropriate and clearly reported in the study? If yes, +1 point. If no, all points deleted.

(3) Were the reasons for patient withdrawals and dropouts described, for each treatment group? If yes, +1 point.

A separate scoring for quality assessment was obtained and independently assessed by two reviewers (MS, FP) to establish whether the study met the quality criteria in order to reduce the risk of bias. The level of agreement between the two reviewers was calculated using kappa statistics.

3.1.3. Data Extraction. Two reviewers (MS and FP) independently using specially designed data extraction forms extracted the necessary data. Any disagreement was discussed, and a third reviewer (MA) was consulted when necessary.

Authors of studies were contacted for clarification or missing information. Data was excluded until further clarification could be available or if an agreement could not be reached. When the results of a study were published more than once or results were detailed in a number of publications, the most complete data set was sought from all sources and included only once.

Using a standard protocol, the following data were collected from the studies:

(i) name of the authors, date of publication, name of the journal, and setting;

(ii) details on the study design;

(iii) sample size (number/gender);

(iv) follow-up (months); 
(v) treated infrabony defects (number), position of the defects (maxillary/mandibular);

(vi) intervention/barrier-augmentation material, soft tissue closure, eventual antibiotic intake, reassessment;

(vii) control group: intervention/barrier-augmentation material;

(viii) clinical attachment level gain (CALgain);

(ix) pocket probing depth reduction (PPDRed);

(x) recession reduction (RECRed).

3.1.4. Heterogeneity Assessment. The statistical heterogeneity among studies has been assessed in two different ways: Cochran's Q statistical test [10] and $I^{2}$ test [11] were applied to the selected studies. A fixed-effects model was adopted due to the hypothesis of a population of studies with similar characteristics.

In the case of high heterogeneity values, subgroups, and sensitivity, analysis was performed based on

(i) study site (maxillary/mandibular);

(ii) regenerative material/s used (bone graft and/or resorbable/not resorbable barrier);

(iii) surgical technique used.

3.1.5. Data Synthesis. To summarize and compare studies, data were displayed as a weighted mean difference (WMD) in primary and secondary outcomes. Using this index, data from articles was directly pooled together (means and $95 \% \mathrm{CI}$ ).

(i) For dichotomous outcomes, the estimates were expressed as relative risk ratio (RR) together with 95\% CI.

(ii) For continuous outcomes, standardized mean differences and $95 \%$ confidence intervals were used to summarize the data for each study.

The study-specific estimates were pooled using the fixedeffects model (Woolf's method). If a significant heterogeneity was found, the random effect model result was presented.

Forest plots were created to illustrate the effects of the different studies and the global estimation.

SPSS Statistics ${ }^{\mathrm{TM}}$ software was used to perform all analyses. Statistical significance has been defined as a $p$ value $<0.05$.

3.1.6. Sensitivity Analysis and Bias Detection. Sensitivity analysis was performed excluding each of the studies step by step from the meta-analysis and evaluating the changes in the global estimation.

Publication biases were evaluated using a funnel plot and Egger's linear regression method.

3.1.7. Final Recommendation. A final recommendation will be extracted from the results of this meta-analysis, considering their clinical significance.

\section{Results}

4.1. Study Selection. The search identified 39 articles on a record of 73 further filtered for "Humans," "Clinical Trial," and "English Language." The independent screening of the titles and abstracts led to the rejection of 18 papers. The full text of the remaining 21 papers was then searched. For 1 study, the full text was not obtained [12], so the final pool was 20 studies. Out of these, 3 papers were further rejected for the following reasons: two studies did not provide a control group $[13,14]$ and one was not a fully RCT [15]. Two articles were rejected because they did not provide comprehensive data and/or standard deviations to be analysed $[16,17]$.

On the first screening, agreement between the reviewers was met for all the articles except one [18], because of the lab method for PRP gel preparation. The 3 rd reviewer solved the debate by accepting it.

The final number of included studies was 15, and their characteristics are reported in Table 1 . Not all the considered studies reported the mean change and the SD value for each outcome; in these cases, the studies were excluded from meta-analysis regarding the missing data (Figure 1).

4.1.1. Classification of Studies according to Treatments. The included studies were grouped according to provided treatment. This action allowed us to analyse better the performance of PRP adding in the following test groups:

Group 1: biomodulators versus biomodulators and PRP (2 articles),

Group 2: grafts versus grafts and PRP (6 articles),

Group 3: none versus PRP alone (1 article),

Group 4: grafts and membranes versus grafts, membranes, and PRP (6 articles).

4.2. Methodological Quality of Included Studies. The quality of the included studies was assessed according to Jadad scoring [9].

Scoring was independently assessed by the reviewers, and all the studies reported a minimum of 2 points or above, allowing them to be included.

To test the extent of interagreement between the two reviewers, Cohen's Kappa Statistics was used.

Its value lies between -1 and 1 , where 1 is the perfect agreement, 0 is exactly what would be expected by chance, and negative values indicate agreement less than chance, that is, potential systematic disagreement.

The calculated point estimate of Cohen's kappa statistic $\kappa$ was 0.74 , which according to the commonly cited scale for interpretation of kappa statistic (Landis and Koch [33]) indicates a substantial agreement between the two reviewers. The $Z$ score $=5.77$ with $p$ value $<.0001$ showed that $\kappa$ is different from zero. The $95 \%$ confidence limits for $\kappa$ were $(0.52,0.97)$.

4.3. Heterogeneity Assessment. In order to evaluate if a within-study or between-study variability occurred, heterogeneity was assessed. Cochran's Q test was calculated although the small number of included studies led to the consideration of $I^{2}$ statistics in a fixed-effects model. The $I^{2}$ statistics showed a substantial heterogeneity for VCAL and 


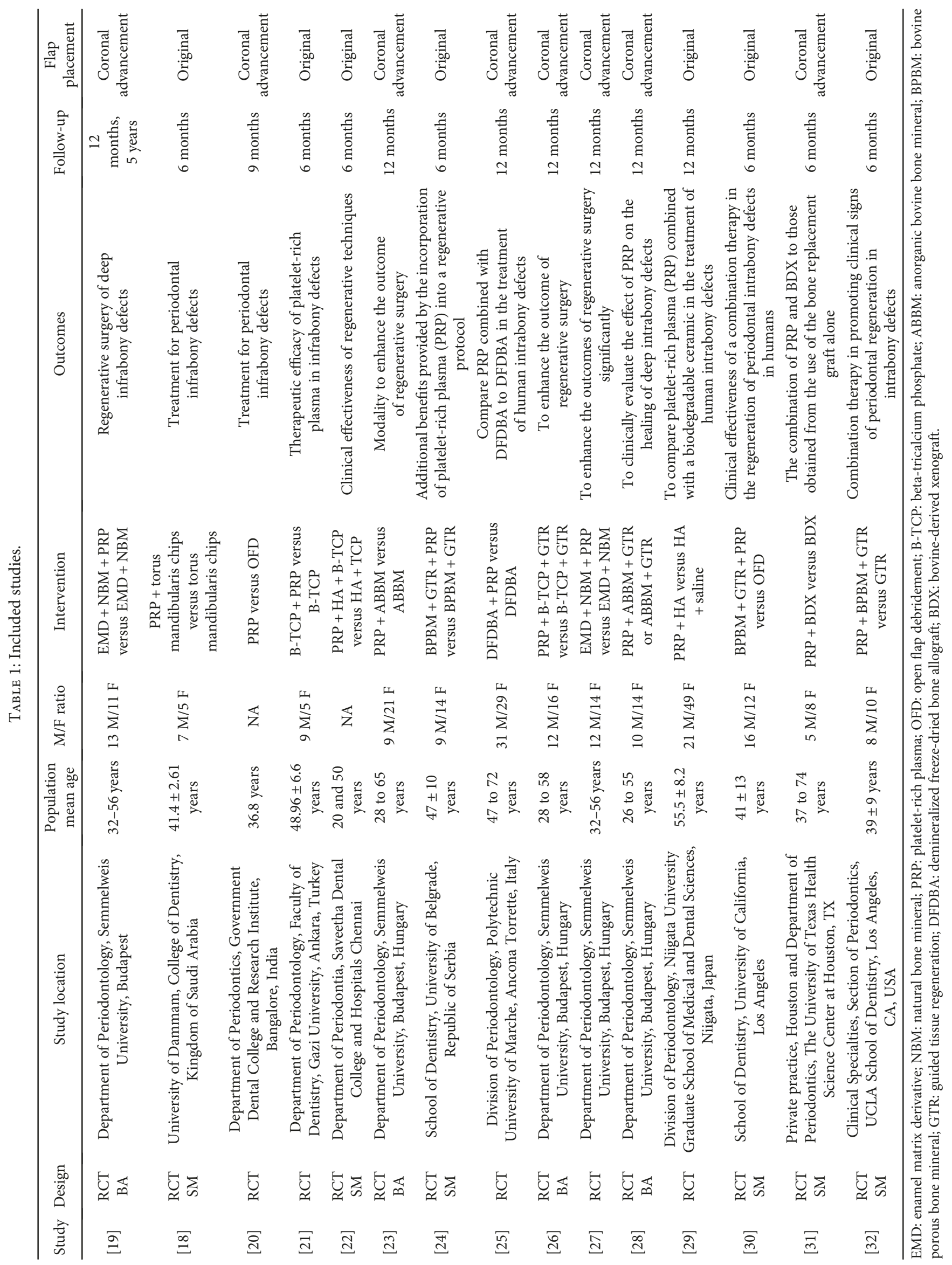




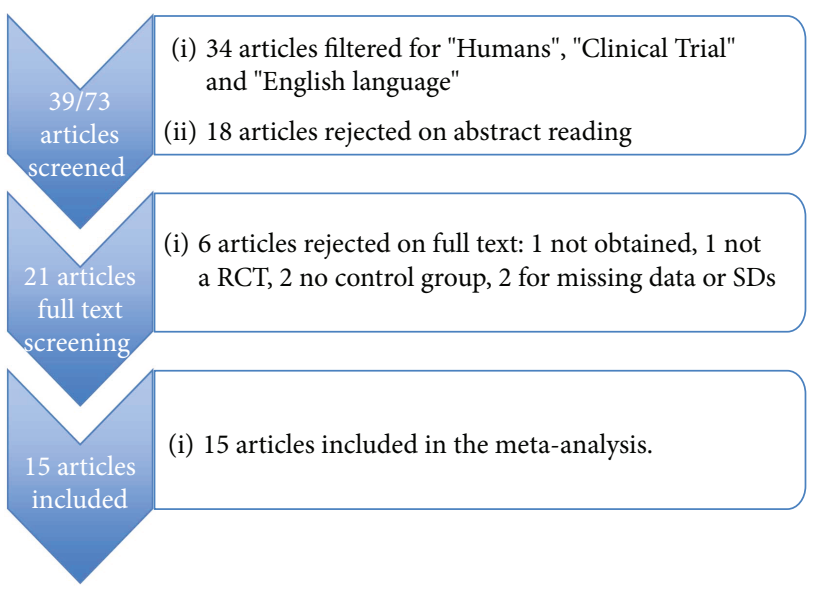

FIGURE 1: Flow chart of the screening process.

VPD outcomes, while no heterogeneity was found according to REC.

\subsection{Change in Vertical Clinical Attachment Level (VCAL)} (Closed Assessment). To test the effectiveness of using PRP in addition to the adopted treatment, 95\% confidence intervals were constructed for difference between the means of test and control groups. The graphical presentation is reported in Figure 2. Out of nine articles, results of five articles led us to accept the hypothesis of no difference between the test and control groups, whereas four articles (Hanna et al. [31], Okuda et al. [29], Piemontese et al. [25], and Kaushick et al. [22]) suggested results in favour of the test group as the SDs showed a mean CAL gain of $2 \mathrm{~mm}$ compared to the control group.

4.5. Change in Vertical Probing Depth (VPD). Twelve articles were able to provide data regarding vertical probing depth (VPD).

Four out of these $12[23,26-28]$ studies showed no difference between the test and control groups, whereas the remaining 8 favoured the addition of PRP showing a VPDRed of about $1.5 \mathrm{~mm}$ (see Figure 3).

4.6. Change in Recession. Out of 8 articles providing useful data for analysis, only one [20] seemed to show the effectiveness of the test group versus the control group in recession reduction of about $0.5 \mathrm{~mm}$ after treatment. The other seven studies did not provide any evaluable difference between groups (see Figure 4).

4.7. Change in Clinical Outcomes Regarding Treatment Groups. According to the categorization of treatments into 4 groups, only 2 of them provided evaluable data regarding the adopted clinical outcomes: grafts + PRP and grafts $+\mathrm{PRP}+$ membranes.

Biomodulators and PRP alone included one single evaluable observation as the other selected articles did not provide any mean baseline-final VCAL, VPD, and REC change or the SD.

When comparing VPD in grafts + PRP and grafts + PRP + membrane, five articles' sample data were available for each of $\mathrm{X}^{-}$grafts $+\mathrm{PRP}$ and $\mathrm{X}^{-}$grafts $+\mathrm{PRP}+$ membrane. The $t$ statistic value $t=4.60$ with $p$ value $<0.0001$ suggested that we may reject null hypothesis in favour of $\mu$ grafts + PRP at $\alpha=5 \%$.

When comparing VCAL in "grafts + PRP" and "grafts + PRP + membrane," two articles' sample data were available for X grafts + PRP and five articles' data for X grafts + PRP + membrane. The $t$ statistic value $t=2.86$ with $p$ value $=0.0045$ suggested that we may reject null hypothesis in favour of $\mu$ grafts + PRP at $\alpha=5 \%$.

When comparing REC in "grafts + PRP" and "Grafts + PRP + membrane," two articles' data were available for computing $\mathrm{X}^{-}$grafts $+\mathrm{PRP}$ whereas four available articles provided values for $\mathrm{X}$ grafts $+\mathrm{PRP}+$ membrane. The $t$ statistic value $t=8.68$ with $p$ value $<0.0001$ again suggested to reject null hypothesis in favour of $\mu$ grafts + PRP at $\alpha=5 \%$.

4.8. Comparison of Clinical Outcomes Regarding the Adopted Surgical Technique. For VCAL, the mean of the test group, that is, "coronally placed" (based on test group of seven articles) was compared with the mean of control group, that is, "original position" (based on test group of two articles).

For VPD, the mean of the test group, that is, "coronally placed" (based on test group of seven articles) is compared with the mean of control group, that is, "original position" (based on test group of five articles).

For VCAL, to test the hypothesis $\mathrm{H} 0, \mu$ coronally placed $=\mu$ original position, $\overline{\mathrm{X}}$ coronally is computed on the basis of seven observations/articles and $\bar{X}$ original is the mean of two observations/articles. The value of $t$ statistic is calculated as $t=0.16$ with $p$ value $=0.3777$. So we may accept the null hypothesis of equality of two means at 0.05 level of significance.

For VPD, to test the hypothesis $\mathrm{H} 0, \mu$ coronally placed $=\mu$ original position, $\mathrm{X}^{-}$coronally is computed on the basis of seven observations/articles and $\mathrm{X}^{-}$original is the mean of five observations/articles. The value of $t$ statistic $t=-1.26$ with $p$ value $=0.2071$ again leads to accept the equality of two means at 0.05 level of significance.

\section{Discussion}

The present systematic review was intended to investigate the controversial results raised from similar papers already published and to update those. The objective was to provide a possible evidence for a better performance of regenerative surgery in infrabony defects with the adjunctive use of autologous blood-derived growth factors as PRP or focusing on the different surgical techniques adopted and subsequently to address future research on the topic.

According to the CONSORT guidelines, our consideration aimed to include only RCTs with a quality assessment equal or more than 2 according to Jadad classification. This allowed us to include only 15 articles from a starting batch of 73 .

Although we followed a strict selection and the quality testing of the included studies, a significant heterogeneity was found, leading us to implement other strategies for categorizations of studies in order to assess and solve it. The 
95\% C.I. of difference of means (test - control) for VCAL

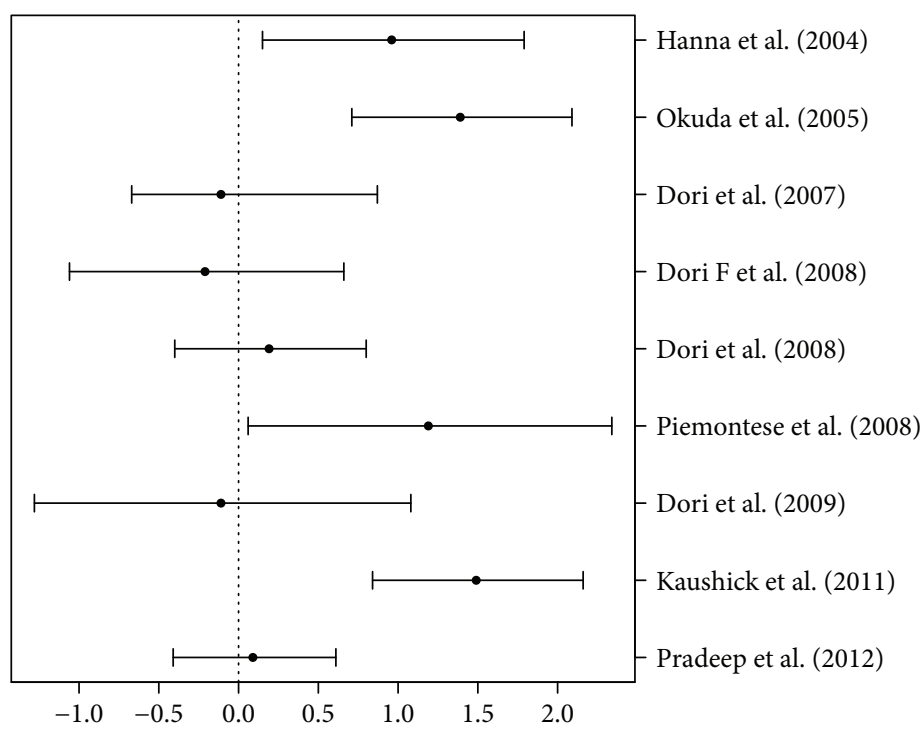

FIGURE 2: 95\% confidence interval presentation for difference between means of test group and control group for VCAL, that is, mean (test) - mean (control).

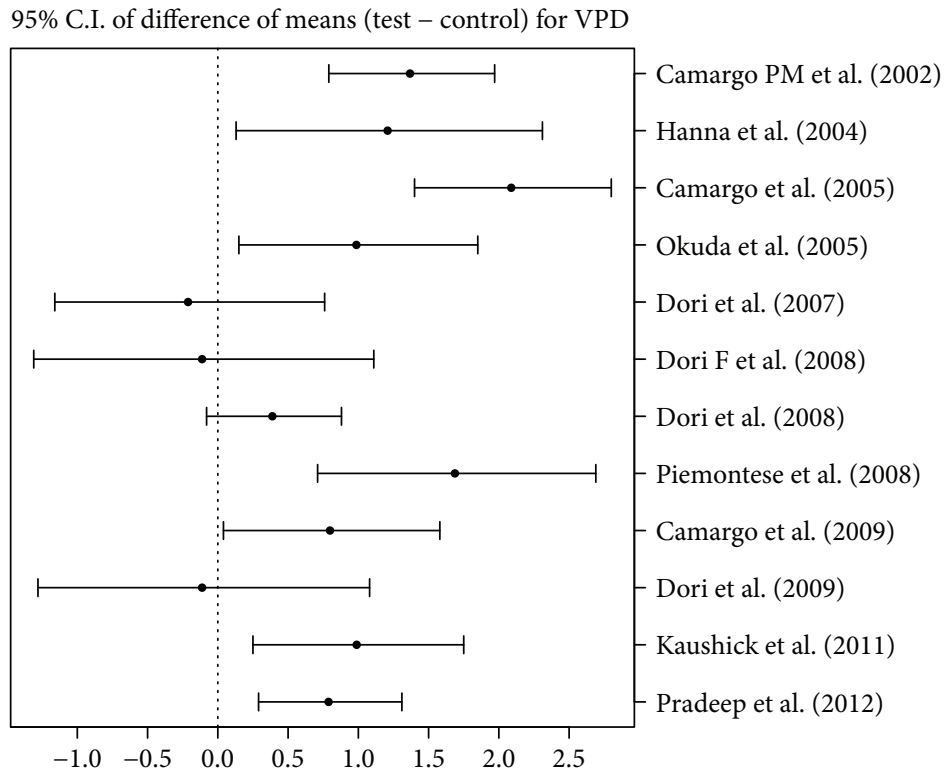

FIGURE 3: 95\% confidence interval presentation for difference between means of test group and control group for VPD, that is, mean (test) - mean (control).

adopted solutions categorized the studies according to the treatment in 4 classes (biomodulators, grafts, grafts + resorbable membranes, and treatment without materials). It also categorized it according to the surgical flap approach (coronally placed or replaced).

Another problem was the high number of the studies that did not provide standard deviations about the provided outcome values. This matter did not allow us to consider them in the meta-analysis due to the need of data regarding the sample variations and of the mean change of the outcomes from baseline examination to the reassessment after the follow-up.
5.1. Overall Intergroup Analysis. The overall meta-analysis performed on the main clinical outcomes (VPD, VCAL, and REC) led us to explore the behaviour of the test group (PRP added) in favouring a better healing in infrabony defect.

The reported $95 \%$ confidence intervals of 4 main studies $[22,25,29,31]$ were presenting favourable results for VPD or VCAL, while not the same for REC. This aspect would suggest an efficient clinical attachment gain inside the infrabony defect due to the adjunctive use of PRP as an appropriate regenerative method. Nevertheless, no radiographic comparison of the alveolar bone levels 




Figure 4: 95\% confidence interval presentation for difference between means of test group and control group for REC, that is, mean (test) - mean (control).

before and after treatment has been reported or systematically assessed, so we cannot provide a precise interpretation about the healing process.

In all the considered studies, the main treatment performed was the combination of a bone graft (HA, DFDBA, and TCP) with PRP without the use of any barrier or a membrane. The performed surgical procedure was flap repositioning without any coronal advancement.

5.2. Intragroup Analysis. When comparing the efficacy of different regenerative materials or techniques among them, the analysis was possible only when considering 2 out of 4 classes of categorization due to missing data.

The combination of bone grafts with PRP was always producing better clinical results in terms of CALgain and pocket reduction than the adjunctive use of membranes after short- and longer term reassessment.

This finding is in line with another systematic review on the same topic [7], which suggested that maybe PRP itself can act as a barrier due to the dense fibrin network produced after platelet activation. The adjunctive use of a membrane either resorbable or not could not allow any interaction between the chemokines and GFs released in the wound area and the overlying connective tissue.

When we looked if the grafting material showed any possible effect, no differences have been identified favouring a specific category. It is therefore suggested that the grafting material is acting as a scaffold leaving the PRP to execute the inductive phase of the healing process.

5.3. Adopted Surgical Technique. According to the evidence of a better performance in CALgain of coronally placed flaps in the regenerative approach to infrabony defects [34], our investigation moved to analyse if the adopted surgical technique or the use of adjunctive PRP could justify the observed better results in the test groups of selected studies. The considered surgical approaches were the replacement and the coronal placement. Due to the assessed high heterogeneity, a categorization according to the technique was performed using the test groups (PRP added) of the included studies. In comparison, the test group was the coronally advanced flap and the control was the replaced flap.

Although not the same number of articles were reporting the use of each one, a " $t$ test" was possible considering only 2 main clinical outcomes: VPD and VCAL.

In all cases, no differences in terms of CAL gain or PPD reduction between the two adopted techniques have been highlighted. The blood clot stability was achieved in both conditions, and the healing process could reasonably happen due to the presence of the growth factors. Even the recession reduction could be outlined with the replaced flap as the evidence in the overall analysis regarding REC outcomes has been suggested [20].

\section{Conclusions}

We conclude from the data in this systematic review that the adjunctive use of PRP in the regenerative treatment of infrabony defects can be considered as an affordable technique to get a better CAL gain and PPD reduction in the surgical treatment of periodontal infrabony defects. Anyway, the limitations of the provided studies are the lack of baseline data regarding the defect size and their morphology, the absence of reports of other relevant clinical outcomes, as the bone fill, and the heterogeneity between studies.

On the basis of this systematic review, the regeneration/ repair of infrabony defects would favour the use of adding PRP to a simple surgical repositioned flap technique, like in the open flap debridement (OFD), with the use of bone grafts (xenografts, HA, or TCP). No better results would be achievable using combinations with biomodulators (Emdogain) or membranes, the PRP just would act as a biomodulator itself. 
In a biological sense, this observation would state for the biomolecular signalling action between PRP and the surrounding cellular environment that any membrane could interrupt or modify. The use of bone grafts would state as a blood clot stabilizer enhancing the osteoinductive properties of the PRP itself.

\section{Future Research/Observations}

According to the main reported pitfalls, future studies should be aimed first, designed according to RCT schemes in order to provide clinical evidences.

A comparison between a surgical flap approach alone and the adjunctive use of PRP would be needful in order to explore the role of growth factors alone in periodontal regeneration and the healing process, as well as the radiographic bone level assessment before and after treatment, as they represent a critical parameter in success assessment.

In order to explore which growth factor would be better suited in periodontal procedures, a multiple-arm RCT would be needful comparing PRP with other blood-derived agents available as well as with the different techniques adopted to deliver it.

\section{Ethical Approval}

This article does not contain any studies with human participants or animals performed by any of the authors.

\section{Conflicts of Interest}

Mubashir Saleem declares that he has no conflict of interest. Flavio Pisani declares that he has no conflict of interest. Faisal Maqbool Zahid declares that he has no conflict of interest. Ioannis Georgakopoulos declares that he has no conflict of interest. Teuta Pustina-Krasniqi declares that she has no conflict of interest. Edit Xhajanka declares that she has no conflict of interest. Maher Almasri declares that he has no conflict of interest.

\section{Authors' Contributions}

Mubashir Saleem and Flavio Pisani contributed equally to this paper.

\section{References}

[1] G. Polimeni, A. V. Xiropaidis, and U. M. E. Wikesjo, "Biology and principles of periodontal wound healing/regeneration," Periodontology 2000, vol. 41, no. 1, pp. 30-47, 2006.

[2] B. L. Eppley, W. S. Pietrzak, and M. Blanton, "Platelet-rich plasma: a review of biology and applications in plastic surgery," Plastic and Reconstructive Surgery, vol. 118, no. 6, pp. 147e-159e, 2006.

[3] S. Kotsovilis, N. Markou, E. Pepelassi, and D. Nikolidakis, "The adjunctive use of platelet-rich plasma in the therapy of periodontal intraosseous defects: a systematic review," Journal of Periodontal Research, vol. 45, no. 3, pp. 428-443, 2010.

[4] N. S. Arora, T. Ramanayake, Y. F. Ren, and G. E. Romanos, "Platelet-rich plasma in sinus augmentation procedures: a systematic literature review: Part II," Implant Dentistry, vol. 19, no. 2, pp. 145-157, 2010.

[5] D. Simon, S. Manuel, V. Geetha, and B. R. Naik, "Potential for osseous regeneration of platelet-rich plasma-a comparative study in mandibular third molar sockets," Indian Journal of Dental Research, vol. 15, no. 4, pp. 133-136, 2004.

[6] A. R. Pradeep, S. K. Shetty, G. Garg, and S. Pai, "Clinical effectiveness of autologous platelet-rich plasma and peptideenhanced bone graft in the treatment of intrabony defects," Journal of Periodontology, vol. 80, no. 1, pp. 62-71, 2009.

[7] M. Del Fabbro, M. Bortolin, S. Taschieri, and R. Weinstein, "Is platelet concentrate advantageous for the surgical treatment of periodontal diseases? A systematic review and meta-analysis," Journal of Periodontology, vol. 82, no. 8, pp. 1100-1111, 2011.

[8] CEBM, "Centre for Evidence-Based Medicine," http://www.ce bm.net/.

[9] A. R. Jadad, R. A. Moore, and D. Carroll, "Assessing the quality of reports of randomized clinical trials: is blinding necessary?," Controlled Clinical Trials, vol. 17, no. 1, pp. 1-12, 1996.

[10] W. G. Cochran, "The combination of estimates from different experiments,” Biometrics, vol. 10, no. 1, pp. 101-129, 1954.

[11] J. P. T. Higgins and S. G. Thompson, "Quantifying heterogeneity in a meta-analysis," Statistics in Medicine, vol. 21, no. 11, pp. 1539-1558, 2002.

[12] X. Y. Ouyang and J. Qiao, "Effect of platelet-rich plasma in the treatment of periodontal infra-bony defects in humans," Chinese Medical Journal, vol. 119, no. 18, pp. 1511-1521, 2006.

[13] D. Moder, F. Taubenhansl, K. A. Hiller, G. Schmalz, and M. Christgau, "Influence of autogenous platelet concentrate on combined GTR/graft therapy in intrabony defects: a 7year follow-up of a randomized prospective clinical splitmouth study," Journal of Clinical Periodontology, vol. 39, no. 5, pp. 457-465, 2012.

[14] A. R. Pradeep, S. Pai, G. Garg, P. Devi, and S. K. Shetty, “A randomized clinical trial of autologous platelet-rich plasma in the treatment of mandibular degree II furcation defects," Journal of Clinical Periodontology, vol. 36, no. 7, pp. 581-588, 2009.

[15] J. Czuryszkiewicz-Cyrana and J. Banach, "Autogenous bone and platelet-rich plasma (PRP) in the treatment of intrabony defects," Advances in Medical Sciences, vol. 51, Supplement 1, pp. 26-30, 2006.

[16] L. Harnack, R. H. Boedeker, I. Kurtulus, S. Boehm, J. Gonzales, and J. Meyle, "Use of platelet-rich plasma in periodontal surgery-a prospective randomised double blind clinical trial," Clinical Oral Investigations, vol. 13, no. 2, pp. 179-187, 2009.

[17] Z. Yassibag-Berkman, O. Tuncer, T. Subasioglu, and A. Kantarci, "Combined use of platelet-rich plasma and bone grafting with or without guided tissue regeneration in the treatment of anterior interproximal defects," Journal of Periodontology, vol. 78, no. 5, pp. 801-809, 2007.

[18] K. S. Hassan, A. S. Alagl, and A. Abdel-Hady, "Torus mandibularis bone chips combined with platelet rich plasma gel for treatment of intrabony osseous defects: clinical and radiographic evaluation," International Journal of Oral \& Maxillofacial Surgery, vol. 41, no. 12, pp. 1519-1526, 2012.

[19] F. Döri, N. Arweiler, T. Húszár, I. Gera, R. J. Miron, and A. Sculean, "Five-year results evaluating the effects of platelet-rich plasma on the healing of intrabony defects treated with enamel matrix derivative and natural bone mineral," Journal of Periodontology, vol. 84, no. 11, pp. 1546-1555, 2013. 
[20] A. R. Pradeep, N. S. Rao, E. Agarwal, P. Bajaj, M. Kumari, and S. B. Naik, "Comparative evaluation of autologous platelet-rich fibrin and platelet-rich plasma in the treatment of 3-wall intrabony defects in chronic periodontitis: a randomized controlled clinical trial," Journal of Periodontology, vol. 83, no. 12, pp. 1499-1507, 2012.

[21] B. Ozdemir and E. Okte, "Treatment of intrabony defects with beta-tricalciumphosphate alone and in combination with platelet-rich plasma," Journal of Biomedical Materials Research Part B:Applied Biomaterials, vol. 100B, no. 4, pp. 976-983, 2012.

[22] B. T. Kaushick, N. D. Jayakumar, O. Padmalatha, and S. Varghese, "Treatment of human periodontal infrabony defects with hydroxyapatite+ $\beta$ tricalcium phosphate bone graft alone and in combination with platelet rich plasma: a randomized clinical trial," Indian Journal of Dental Research, vol. 22, no. 4, pp. 505-510, 2011.

[23] F. Döri, V. Kovács, N. B. Arweiler et al., "Effect of platelet-rich plasma on the healing of intrabony defects treated with an anorganic bovine bone mineral: a pilot study," Journal of Periodontology, vol. 80, no. 10, pp. 1599-1605, 2009.

[24] P. M. Camargo, V. Lekovic, M. Weinlaender, T. DivnicResnik, M. Pavlovic, and E. B. Kenney, "A surgical reentry study on the influence of platelet-rich plasma in enhancing the regenerative effects of bovine porous bone mineral and guided tissue regeneration in the treatment of intrabony defects in humans," Journal of Periodontology, vol. 80, no. 6, pp. 915-923, 2009.

[25] M. Piemontese, S. D. Aspriello, C. Rubini, L. Ferrante, and M. Procaccini, "Treatment of periodontal intrabony defects with demineralized freeze-dried bone allograft in combination with platelet-rich plasma: a comparative clinical trial," Journal of Periodontology, vol. 79, no. 5, pp. 802-810, 2008.

[26] F. Döri, T. Huszár, D. Nikolidakis et al., "Effect of platelet-rich plasma on the healing of intrabony defects treated with Beta tricalcium phosphate and expanded polytetrafluoroethylene membranes," Journal of Periodontology, vol. 79, no. 4, pp. 660-669, 2008.

[27] F. Döri, D. Nikolidakis, T. Húszár, N. B. Arweiler, I. Gera, and A. Sculean, "Effect of platelet-rich plasma on the healing of intrabony defects treated with an enamel matrix protein derivative and a natural bone mineral," Journal of Clinical Periodontology, vol. 35, no. 1, pp. 44-50, 2008.

[28] F. Döri, T. Huszár, D. Nikolidakis, N. B. Arweiler, I. Gera, and A. Sculean, "Effect of platelet-rich plasma on the healing of intrabony defects treated with an anorganic bovine bone mineral and expanded polytetrafluoroethylene membranes," Journal of Periodontology, vol. 78, no. 6, pp. 983-990, 2007.

[29] K. Okuda, H. Tai, K. Tanabe et al., "Platelet-rich plasma combined with a porous hydroxyapatite graft for the treatment of intrabony periodontal defects in humans: a comparative controlled clinical study," Journal of Periodontology, vol. 76, no. 6, pp. 890-898, 2005.

[30] P. M. Camargo, V. Lekovic, M. Weinlaender, N. Vasilic, M. Madzarevic, and E. B. Kenney, "A reentry study on the use of bovine porous bone mineral, GTR, and platelet-rich plasma in the regenerative treatment of intrabony defects in humans," International Journal of Periodontics \& Restorative Dentistry, vol. 25, no. 1, pp. 49-59, 2005.

[31] R. Hanna, P. M. Trejo, and R. L. Weltman, "Treatment of intrabony defects with bovine-derived xenograft alone and in combination with platelet-rich plasma: a randomized clinical trial," Journal of Periodontology, vol. 75, no. 12, pp. 1668-1677, 2004.

[32] P. M. Camargo, V. Lekovic, M. Weinlaender, N. Vasilic, M. Madzarevic, and E. B. Kenney, "Platelet-rich plasma and bovine porous bone mineral combined with guided tissue regeneration in the treatment of intrabony defects in humans," Journal of Periodontal Research, vol. 37, no. 4, pp. 300-306, 2002.

[33] J. R. Landis and G. G. Koch, "The measurement of observer agreement for categorical data," Biometrics, vol. 33, no. 1, pp. 159-174, 1977.

[34] G. Zucchelli and M. De Sanctis, "A novel approach to minimizing gingival recession in the treatment of vertical bony defects," Journal of Periodontology, vol. 79, no. 3, pp. 567-574, 2008. 


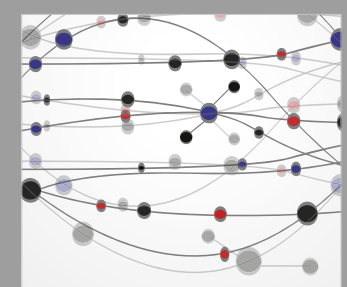

The Scientific World Journal
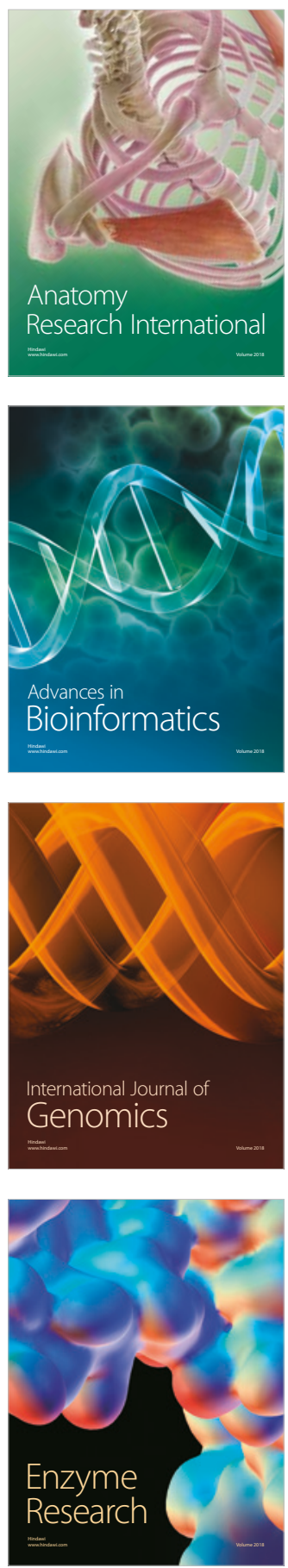
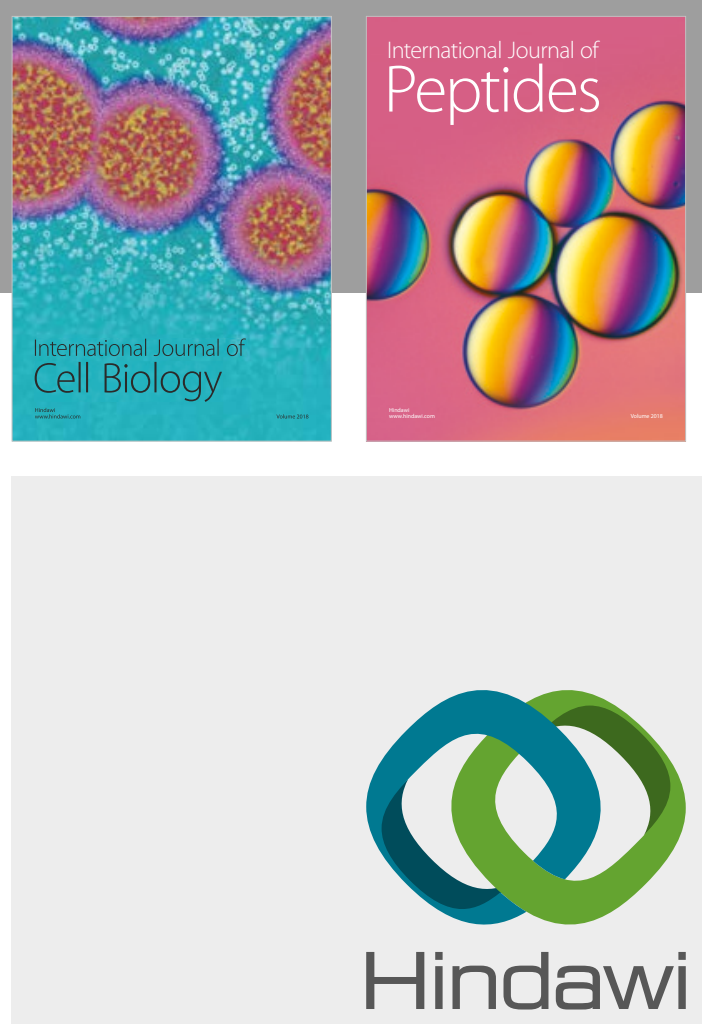

Submit your manuscripts at

www.hindawi.com
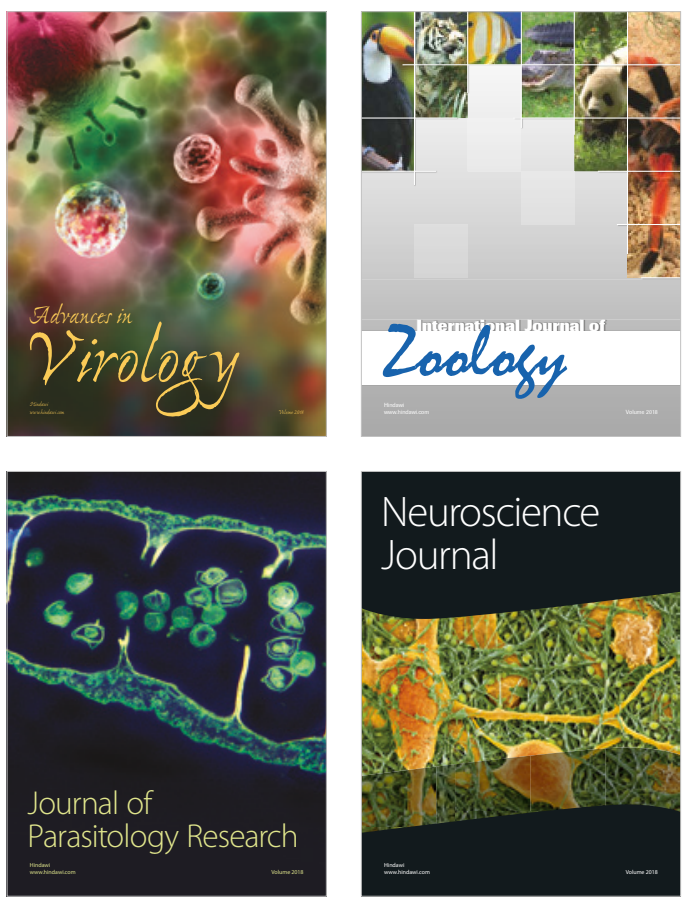
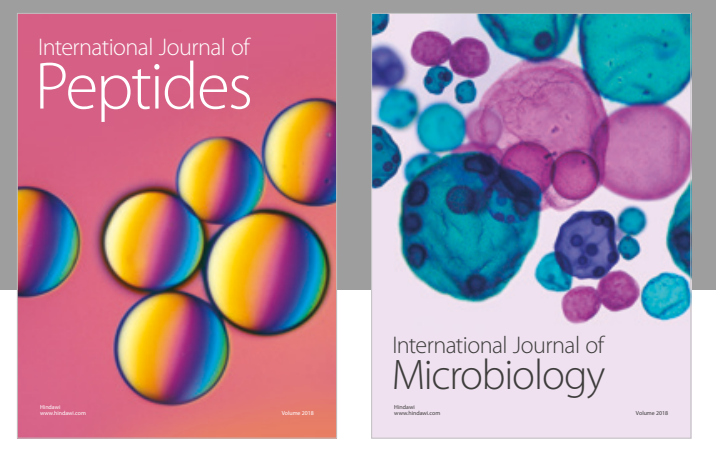

nternational Journal of Microbiology
Journal of
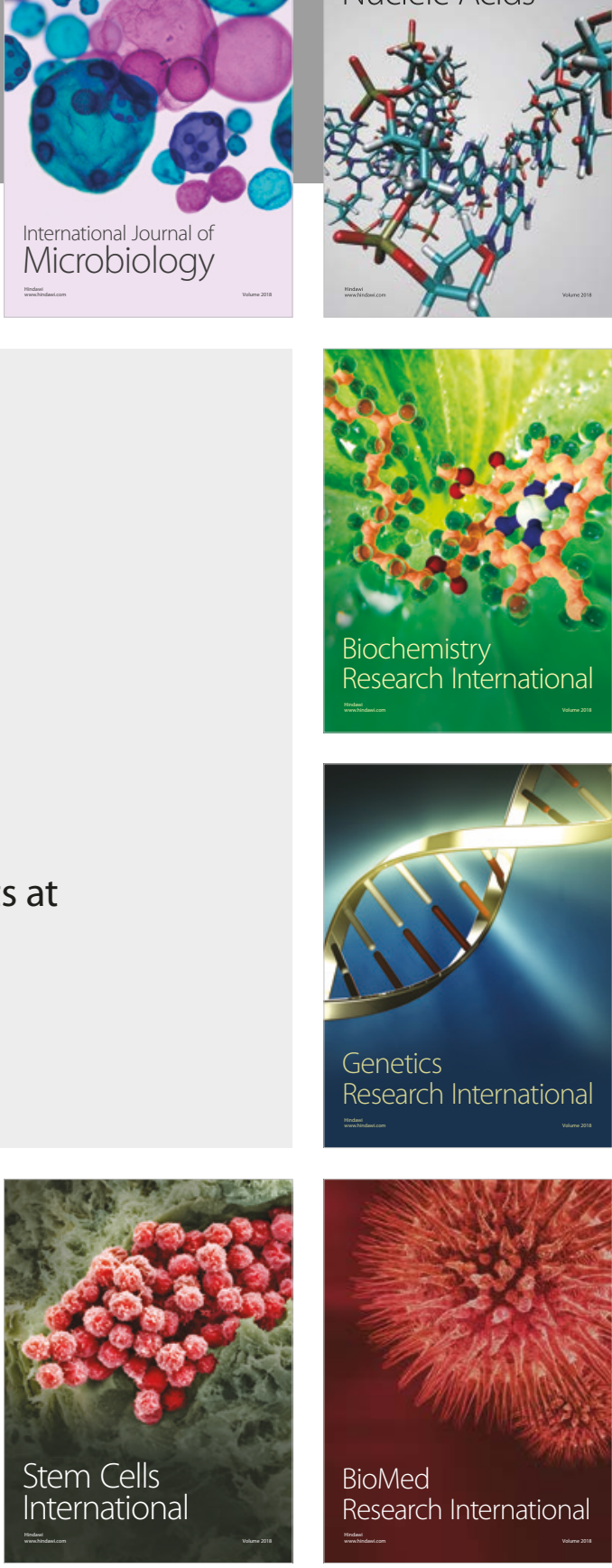
